\title{
Médiévales
}

Langues, Textes, Histoire

81 | automne 2021

Voix laïques (XIe-XIIIe siècle)

\section{André-Yves Bourgès, Le Dossier littéraire de saint Goëznou et la controverse sur la datation de la Vita sancti Goeznovei, suivi en annexe de la vita de saint Ténénan}

Morlaix, Les Lettres Morlaisiennes, 2020, 248 p.

\section{Alban Gautier}

\section{OpenEdition Journals}

Édition électronique

URL : https://journals.openedition.org/medievales/11950

DOI : 10.4000/medievales. 11950

ISSN : $1777-5892$

\section{Éditeur}

Presses universitaires de Vincennes

\section{Édition imprimée}

Date de publication : 12 janvier 2022

Pagination : 197-199

ISBN : 978-2-37924-202-1

ISSN : 0751-2708

\section{Référence électronique}

Alban Gautier, « André-Yves Bourgès, Le Dossier littéraire de saint Goëznou et la controverse sur la datation de la Vita sancti Goeznovei, suivi en annexe de la vita de saint Ténénan », Médiévales [En ligne], 81 | automne 2021, mis en ligne le 31 janvier 2022, consulté le 22 avril 2022. URL : http:// journals.openedition.org/medievales/11950; DOI : https://doi.org/10.4000/medievales.11950

Ce document a été généré automatiquement le 22 avril 2022

Tous droits réservés 


\section{André-Yves Bourgès, Le Dossier littéraire de saint Goëznou et la controverse sur la datation de la Vita sancti Goeznovei, suivi en annexe de la vita de saint Ténénan}

Morlaix, Les Lettres Morlaisiennes, 2020, 248 p.

Alban Gautier

\section{RÉFÉRENCE}

André-Yves Bourgès, Le Dossier littéraire de saint Goëznou et la controverse sur la datation de la Vita sancti Goeznovei, suivi en annexe de la vita de saint Ténénan, Morlaix, Les Lettres Morlaisiennes, 2020, 248 p.

1 La Vie de Goëznou, un saint monastique relativement obscur du diocèse breton de Léon, aurait sans nul doute figuré au nombre des textes mineurs, peu étudiés et par la seule érudition locale, sans la longue et âpre controverse que sa datation a suscitée - une controverse entamée dès le milieu du XIX ${ }^{e}$ siècle, qui a connu des rebondissements jusqu'à nos jours, principalement parce qu'elle est porteuse de questionnements et d'hypothèses touchant à des sujets aussi vastes que la circulation des textes et des récits dans le monde brittonique et anglo-normand, les sources de la légende arthurienne et en particulier de l'œuvre de Geoffroy de Monmouth, le peuplement de la Bretagne au haut Moyen Âge, et même l'historicité du roi Arthur - rien de moins !

De cette Vie aujourd'hui perdue, il ne reste que des extraits, copiés pour eux-mêmes ou intégrés à d'autres textes à la fin du Moyen Âge, ainsi que des résumés effectués par des érudits de l'époque moderne. A.-Y. Bourgès en identifie six sur une période d'un peu plus de trois siècles, quatre en latin, deux en français : 1- les 231 vers conservés du 
poème intitulé Livre des faits d'Arthur (début du XIV ${ }^{e}$ siècle) ; 2- des extraits insérés dans la Chronique de Saint-Brieuc (fin XIV ${ }^{e}$-début $\mathrm{XV}^{\mathrm{e}}$ siècle) ; 3-des passages copiés dans la seconde moitié $\mathrm{du} x \mathrm{v}^{\mathrm{e}}$ siècle dans un manuscrit connu sous le nom de Vetus collectio (Rennes, AD Ille-et-Vilaine, ms. 1F 1003); 4- les résumés en français inclus dans deux versions successives (v. 1475-1480 et v. 1500-1505) des Chroniques de Pierre Le Baud ; 5la notice sur Goëznou dans un bréviaire diocésain de l'évêché de Léon publié en 1516; 6- le compte rendu détaillé en français qu'en donne le dominicain Albert Le Grand dans sa célèbre collection de vies de saints bretons parue en 1637.

Dans une section intitulée « corpus » (p. 103-147), A.-Y. Bourgès offre des transcriptions de cinq de ces six témoins, accompagnées de traductions françaises des textes latins. Le seul à ne pas être transcrit est le Livre des faits d'Arthur; on peut regretter cette omission, peut-être due au fait qu'une Américaine en préparerait l'édition. C'est donc en s'appuyant sur ce corpus que l'auteur, à défaut de proposer une édition de la Vie perdue - entreprise par trop périlleuse à laquelle il convenait en effet de renoncer -, fournit un « conspect » de l'œuvre (p. 149-218), à savoir une tentative de reconstitution la plus détaillée possible, sous forme de description du contenu plutôt que d'établissement d'un texte unifié. Abondamment pourvu de notes (linguistiques, philologiques, historiques, topographiques, etc.), ce conspect constitue une solution alternative très convaincante à une édition impossible. Reprenant la structure du compte rendu d'Albert Le Grand, qui constitue la dérivation de l'œuvre originale la plus détaillée, l'auteur s'efforce d'y resituer les extraits latins connus par les autres témoins. Il nous offre ainsi un outil de travail précieux et un descriptif minutieux, nécessairement hypothétique, mais éminemment plausible, qui, sans refermer le dossier, constituera un passage obligé pour toute future étude de la Vita Goeznovei et des innombrables débats qui l'entourent.

On regrettera cependant que l'auteur n'ait pas toujours facilité la tâche de son lecteur et se soit ingénié à disperser des informations qui auraient pu être réunies de manière plus commode. Ainsi, il faut lire attentivement la note 33 de la page 24 pour comprendre que l'item $\mathrm{n}^{\circ} 5$ dans la liste de la page 19 (le bréviaire diocésain de 1516 auquel a travaillé le chanoine Hamon Barbier) correspond au deuxième texte du corpus, transcrit en page 116-121 (l'extrait intitulé De S. Goeznoveo dans le manuscrit français 22321 de la Bibliothèque nationale de France, témoin d'un extrait perdu dans l'édition lacunaire de 1516). Une annonce claire, dès le début de l'introduction, des équivalences entre d'une part les auteurs qui ont eu le texte de la Vie en mains et en ont proposé des extraits en latin ou un résumé en français, et d'autre part les textes transcrits dans le corpus, aurait grandement amélioré le confort de lecture - d'autant plus que la table des abréviations insérée en début d'ouvrage ne recense que les acronymes modernes (titres de revues, noms d'universités ou de centres de recherche); le lecteur ne trouve donc pas de liste des abréviations des textes médiévaux ou modernes étudiés par l'auteur (LFA pour Livre des faits d'Arthur, CB pour Chronicon Briocense, etc.). Enfin, il n'aurait pas été superflu d'indiquer dès leur première mention la langue dans laquelle chacun des textes nous est parvenu : il a fallu quelques longues minutes au présent recenseur, et pas mal de pages tournées et retournées, pour comprendre que le Livre des faits d'Arthur est un poème en hexamètres latins !

Mais venons-en au contenu de l'œuvre et à la controverse qu'elle a suscitée. Il s'agissait d'une Vie relativement brève : la version qu'en a donnée Albert Le Grand tient ici en huit pages; les extraits de la Vetus collectio (qui fournit le texte latin le plus développé) 
en six pages. Après une dédicace et un prologue pseudo-historique qui ont fait couler beaucoup d'encre, ce récit racontait la vie de Goëznou, fondateur d'un monastère au diocèse de Léon, ses rapports avec les pouvoirs locaux et la manière dont le saint avait délimité la clôture de son monastère et y avait défendu le droit d'asile; élu évêque de Léon en 650 , Goëznou serait mort en 675 des mains d'un ouvrier mécontent sur le chantier de l'abbatiale de Quimperlé; l'œuvre s'achevait sur un épilogue évoquant diverses reliques présentes en Bretagne et ailleurs. Émaillée de quelques miracles, de détails plus fournis concernant les reliques et de nombreuses notations topographiques, la Vie mettait certes l'accent sur les vertus du saint, mais soulignait surtout son action de bâtisseur et de défenseur des libertés ecclésiastiques face aux empiétements des pouvoirs laïcs - en d'autres termes, la Vie assez ordinaire d'un saint d'intérêt local.

Or, malgré ce peu d'originalité, l'œuvre s'est retrouvée au centre de débats acharnés portant sur sa datation et sur les conséquences qu'il conviendrait d'en tirer. Cette longue controverse s'est principalement concentrée sur la dédicace et le prologue, lus ou non à la lumière de l'épilogue. En effet, depuis qu'en 1862 Arthur de La Borderie a fait connaître l'existence de la Vetus collectio ( $\mathrm{xv}^{\mathrm{e}}$ siècle), on sait que le texte transmis dans ce manuscrit commence par une dédicace où l'auteur, qui dit s'appeler Guillaume, affirme avoir composé son œuvre en l'an 1019 pour le compte d'un évêque nommé Eudon. Or, le prologue qui suit mentionne plusieurs figures et événements dont le récit n'est nulle part attesté avant l'Historia regum Britanniae de Geoffroy de Monmouth, achevée dans la seconde moitié des années 1130 : on y trouve ainsi le nom de Corineus, la migration des Bretons sur le continent sous la direction de Conan Mériadec, ou encore les «nombreuses victoires en Bretagne et en Gaule» du roi Arthur, toutes choses que Guillaume affirme avoir lues dans une historia britannica.

7 Ainsi, de deux choses l'une: soit la date de 1019 est erronée et l'historia britannica utilisée par Guillaume n'est autre que l'Historia de Geoffroy, soit la date est exacte et nous avons ici la preuve, unique et précieuse, que Geoffroy n'a pas «tout inventé », qu'il a puisé à une tradition qui circulait anciennement en Armorique, voire que le fameux liber vetustissimus britannici sermonis dont il prétend avoir fait usage n'est autre qu'une version vernaculaire (perdue, bien entendu) de la vita Goeznovei. Une fois écartée l'hypothèse d'une interpolation, rendue peu plausible en raison d'une seconde mention de l'évêque Eudon dans l'épilogue, mais aussi de la présence des mêmes éléments dans deux autres témoins (les extraits insérés dans le Chronicon Briocense et dans les Chroniques de Le Baud), il faut bien qu'une de ces deux hypothèses soit la bonne. Le débat n'a guère cessé depuis le milieu du XIx ${ }^{e}$ siècle, faisant s'affronter certains des plus grands spécialistes de la Bretagne médiévale: du côté de la datation haute et de l'ancienneté de la tradition, on trouve bien sûr La Borderie (qui malheureusement n'a jamais proposé d'édition honnête et satisfaisante de la Vetus collectio), mais aussi La Villemarqué, puis Léon Fleuriot et surtout Gwenaël Le Duc, infatigable défenseur de l'authenticité de la datation; du côté des sceptiques, on trouve dès l'origine Gaston Paris et Joseph Loth, puis Edmond Faral et J. S. P. Tatlock, Hubert Guillotel et, enfin, A.Y. Bourgès lui-même.

8 En effet, ce que montre l'auteur de ce livre, c'est que de très nombreuses raisons empêchent d'admettre une date de composition en 1019. En premier lieu, le manuscrit de la Vetus collectio est raturé: le scribe $d u \mathrm{xv}^{e}$ siècle avait commencé par écrire « 1099 » avant de se raviser. Selon A.-Y. Bourgès, la source portait probablement la date 
de 1199, congruente à la quasi-totalité des autres éléments de critique interne et externe de l'œuvre. Parmi ceux qui sont ici mis en avant, on citera l'existence bien attestée d'un évêque Eudon en Léon à la fin $\mathrm{du}_{\mathrm{XII}}{ }^{\mathrm{e}}$ siècle (la liste épiscopale du haut Moyen Âge est en revanche très incertaine), la mention de reliques conservées «au monastère de Châge à Meaux » (une communauté victorine fondée en 1135), le propos général de l'œuvre à lire au prisme de la mise en œuvre de la réforme grégorienne dans la Bretagne du XII ${ }^{e}$ siècle... sans oublier l'insertion de tous les éléments liés à l'Historia regum Britanniae, que l'on explique tout de même bien mieux soixante ans après Geoffroy que cent vingt ans avant lui! Cette datation à la toute fin $\mathrm{du} \mathrm{XII}^{\mathrm{e}}$ siècle serait donc compatible avec une hypothèse séduisante que l'auteur avait émise dans diverses publications antérieures où il proposait d'identifier l'auteur de la Vie de Goëznou avec le plus célèbre des écrivains léonards du Moyen Âge : Guillaume le Breton, l'auteur de la Philippide.

9 Au risque d'attrister tous les enthousiastes de l'historicité de la «tradition bretonne » et de l'authenticité foncière de l'œuvre de Geoffroy de Monmouth, et même si l'on choisit de réserver son jugement à propos de telle ou telle proposition du " conspect » ou de l'identification avec Guillaume le Breton, il faut bien admettre qu'A.-Y. Bourgès a enfoncé un clou de plus - le dernier? - dans le cercueil de la datation haute de la vita Goeznovei et, partant, de la circulation ancienne en Armorique de récits annonçant l'Historia regum Britanniae. Pour contrer sa démonstration méthodique, les éventuels contradicteurs devront faire preuve de beaucoup d'ingéniosité... ou de mauvaise foi.

\section{AUTEURS}

\section{ALBAN GAUTIER}

Université de Caen Normandie, Centre Michel de Boüard CRAHAM UMR 6273 